\title{
Epidemiological, Clinical and Outcome Aspects of Patients with Histoplasmosis associated to HIV infection
}

\author{
Mario León Silva Vergara ${ }^{1}$; Alessandro Henrique Damasceno Escoura Júnior ${ }^{1}$; Delio José Mora1; Renata \\ Margarida Etchebehere ${ }^{2}$; Antônio Carlos Oliveira de Meneses²; Adilha Misson Rua Micheletti². \\ ${ }^{1}$ Infectous diseases unit, Federal University of Triângulo Mineiro, Uberaba, Minas Gerais, Brazil; ${ }^{2}$ Pathology Department, Federal University of Triângulo \\ Mineiro, Uberaba, Minas Gerais, Brazil;
}

\section{INTRODUCTION}

Classically, histoplasmosis was considered an endemic mycosis, until the advent of AIDS era, when it was included as a defining disease of this syndrome. It occurs in approximately $5 \%$ of HIVinfected patients from endemic areas and often evolves as disseminated infection and poor outcome, especially in limited resource settings. This report aims to present epidemiological, clinical and outcome aspects of patients with histoplasmosis associated to HIV-infection admitted in a teaching hospital from 2003 to 2016, including the post mortem findings of those who were submitted to necropsy from 1990 to 2016 .

\section{METHODS}

Medical and diagnosis records of HIV-Infected patients with histoplasmosis confirmed by culture were reviewed. Necropsy records were also reviewed in order to register the main epidemiological, clinical and outcome aspects.

\section{RESULTS}

During the last 13 years, 30 HIV-infected patients with histoplasmosis were admitted. Of these, $19(63.33 \%)$ were male, median age of 35.43 years. Of 20 patients with available data, 18 presented CD4 count $<100$ cells and high viral load values. Fifteen $(50 \%)$ were diagnosed with AIDS and histoplasmosis in the same year, whereas 11 presented histoplasmosis as the first opportunistic infection. The median time between the onset of symptoms and the diagnosis was 32 days while the median hospital stay length was 23 days. Overall $13(43.33 \%)$ patients were cured, $16(53.33 \%)$ died and 1 (3.33\%) lost follow-up.

\section{Clinical Findings in 30 HIV-infected Patients with Histoplasmosis}

Cutaneous lesions

Vomits

Diarrhea

Dyspnea

Hyporexia

Weight Loss

Cough

Hepatoesplenomegaly

Asthenia

Fever
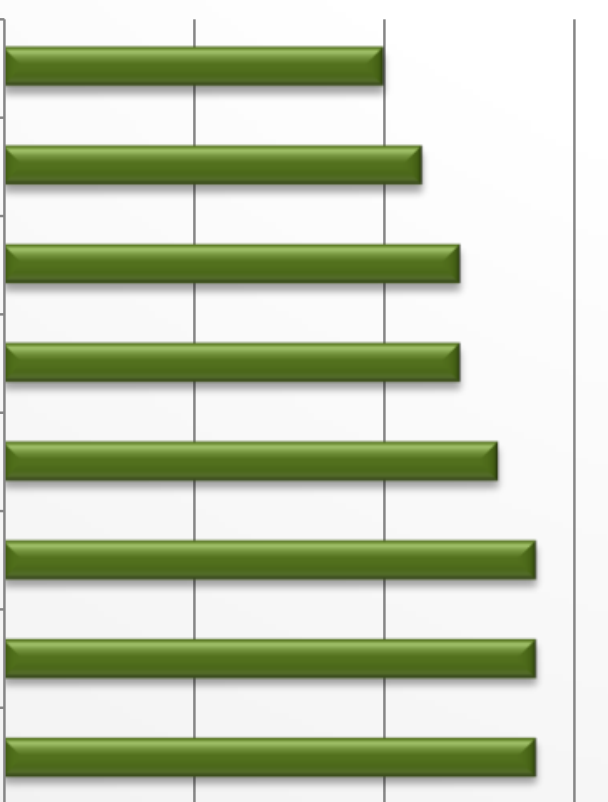

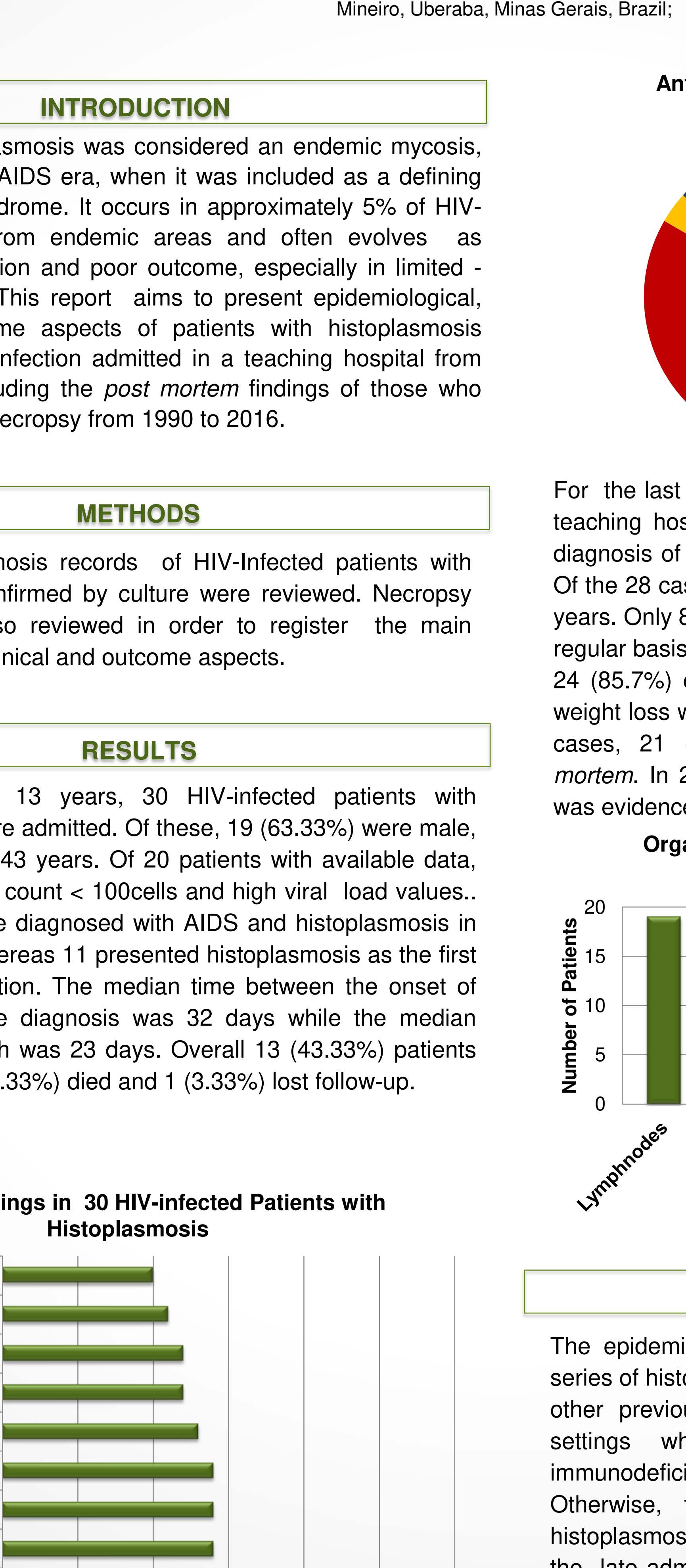
often, clinical and laboratory similarities with other diseases. hospitals in order to evaluate and improve the quality of Clinical diagnosis as well. late admission associated to operational difficulties and Thus, the necropsy exam is remarkable in the teaching

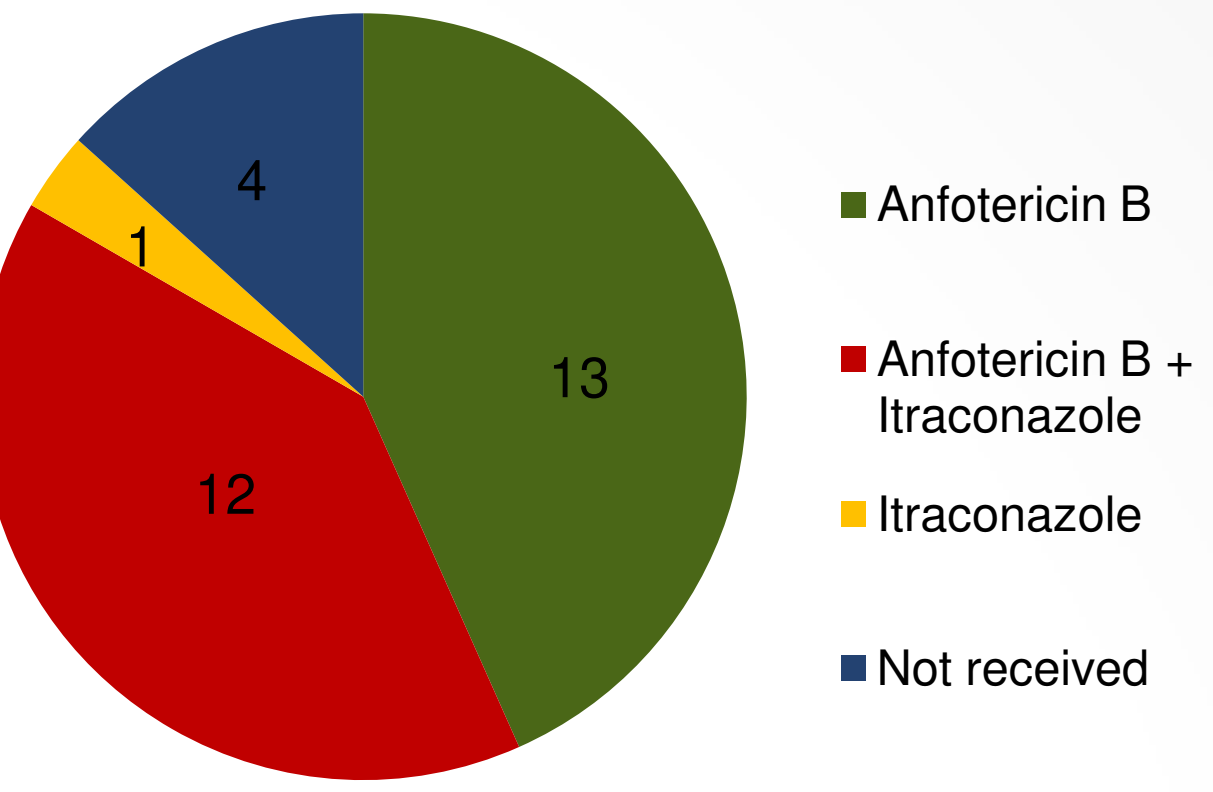
individuals

\section{CONCLUSION}

E-mail:marioleon.dip@mednet.com.br 